\title{
Rule-based semi-automated approach for detection of landslides induced by 18 September 2011 Sikkim, Himalaya, earthquake using IRS LISS3 satellite images
}

\begin{abstract}
Landslide is considered as one of the most devastating and most costly natural hazards in highlands, which is triggered mainly by rainfalls or earthquakes. In comparison with other methods, landslide mapping and monitoring via remote sensing data products are considered as the least expensive method of data collection. The current research attempts to detect landslides which occurred due to a 6.9 magnitude earthquake in Sikkim Himalaya, India, on 18 September 2011 and also to establish the spatial relationship between landslides and the slope of the terrain. To detect the landslides, decision tree method was applied on two Indian remote sensing satellites linear imaging self-scanning sensor (LISS III) images acquired from 2007 and 2011 which were taken before and after the earthquake. As the study area was relatively huge for identifying the landslides, the region was separated into two parts: "tested study area" and "real study area". The overall accuracy of landslide detection was $76 \%$, and $75 \%$ for tested and real study area, respectively. Then, the spatial relationship between the landslides and the slope of the terrain was conducted using the digital elevation model. The results revealed that most of the landslides occurred between the slope of $25^{\circ}$ and $45^{\circ}$ covering $2.3 \mathrm{~km} 2$ and no landslide recorded in the slope of $65^{\circ}-90^{\circ}$ in the real study area. The results obtained in this study may be useful for decision-making and policy support towards reconstruction effort after the landslide occurrence. In addition, the information can be useful for reducing the risk of potential damages to substructures and properties by developing new and efficient strategies.
\end{abstract}

Keyword: Landslides detection; Sikkim; Himalaya; Earthquake; IRS LISS3 satellite images 